\title{
Modelado y optimización de la operación de un sistema de bombeo de múltiples depósitos
}

\author{
Roberto Sanchis e Ignacio Peñarrocha \\ D. Enginyeria de Sistemes Industrials i Disseny, University Jaume I, Castelló, Spain, \\ rsanchis@uji.es, ipenarro@uji.es
}

\section{Resumen}

Este trabajo estudia la optimización de la operación de un sistema de bombeo para suministro de agua por medio de solvers estándar. El sistema consta de varios tanques que abastecen de agua a varios distritos de una ciudad. Cada depósito puede ser llenado desde varios pozos a través de un sistema hidráulico que puede ser reconfigurado por medio de varias válvulas. La operación automatizada del sistema trata de determinar qué válvulas y bombas deben estar activas en cada instante para minimizar el coste de operación, teniendo en cuenta los periodos de tarificación. Se propone un modelo matemático del problema con el fin de formular, en forma matricial, el índice de coste y las restricciones, para poder utilizar solvers estándar como Mosek o CBC. Se trata de un problema de optimización mixta entera, que tiene un coste computacional elevado. Para reducir el coste se propone reducir el número de variables enteras, sustituyéndolas por variables reales a partir de un instante determinado en el horizonte de optimización. Palabras clave: optimización de bombeo, tarifa eléctrica, programación de bombeo.

\section{INTRODUCCIÓN}

Un coste importante en el funcionamiento de los sistemas de abastecimiento de agua es el coste energético asociado al sistema de bombeo. Los consumidores reciben el agua de los tanques de abastecimiento que se llenan de los pozos. El coste de operación depende de los instantes durante el día en que las bombas están funcionando, ya que la tarifa eléctrica varía a lo largo del día, siguiendo periodos predefinidos de tarificación.

El trabajo [4] presenta una revisión de diferentes enfoques para la optimización de sistemas de bombeo de agua, que dependen de la configuración del sistema y de los modelos matemáticos utilizados. Para el problema del llenado de los tanques principales, que es el objetivo de este trabajo, el modelo más comúnmente usado es el balance de masas. En la mayoría de los trabajos, la función de cos- te toma en cuenta la energía consumida, pero no los periodos de tarificación cuando se consume esa energía. Por otro lado, las variables de decisión son usualmente la fracción del tiempo de operación de cada bomba en algunos intervalos predefinidos. También se han propuesto otras variables indirectas de decisión (como los volúmenes de los tanques), pero la relación con el índice de coste y la decisión final (las órdenes a las bombas y válvulas) es demasiado compleja para el caso que estamos estudiando.

En un trabajo más reciente, [1], se revisan diferentes enfoques, centrándose en las tecnologías de optimización dinámica en tiempo real para mejorar la eficiencia energética. Este trabajo describe un software de optimización comercial para sistemas de distribución de agua que puede resolver el problema que abordamos en este trabajo y otros más complejos, pero no se dan los detalles sobre los algoritmos utilizados.

La mayoría de los trabajos recientes, como [6], [7] o [2], utilizan modelos complejos de los sistemas hidráulicos y se basan en complejos algoritmos de optimización, como algoritmos genéticos, "particle swarm" o "simulated annealing". Esos enfoques tienen un coste computacional muy elevado, especialmente para sistemas grandes.

En [5], se utiliza un enfoque de programación lineal para la optimización del funcionamiento de las bombas, pero sólo es aplicable para un sistema de tanque único.

En [3] se presentan tres formulaciones explícitas diferentes del problema de programación óptima de la bomba. Tiene en cuenta la tarifa eléctrica, pero las variables de decisión definen los tiempos de arranque y parada de las diferentes bombas del sistema. El problema de optimización resultante tiene que ser resuelto por algoritmos no lineales, algoritmos genéticos o algoritmos ad hoc semiheurísticos. Esta formulación explícita discreta no es aplicable directamente si el sistema tiene algunas válvulas que pueden reconfigurar la red. En ese caso no es suficiente decidir qué bombas se arrancan o paran en ese momento, sino también la conmutación de las válvulas, que cambia el flujo de 
entrada resultante a los tanques para un estado de bombas dado. Además, nuestro objetivo es utilizar solucionadores estándar para tratar el problema de optimización. Por este motivo, en este trabajo, las variables de decisión son explícitas, pero consisten en la combinación de bombas y válvulas que deben estar activas en cada instante, a partir del conjunto de posibles combinaciones. La formulación matemática propuesta permite utilizar parsers estándar (como Yalmip) y solvers estándar (como CBC), para resolver el problema de optimización.

En la sección II se describe el problema de la operación óptima del sistema de bombeo. En la sección III se desarrolla el modelo matemático del problema. En la sección IV se formula el problema básico de optimización. Un problema de optimización más complejo se formula en la sección V para llegar a soluciones más prácticas, con un número reducido de conmutaciones de la bomba. La sección VI muestra la aplicación a un sistema de bombeo real y la sección VII resume las principales conclusiones.

\section{DESCRIPCIÓN DEL PROBLEMA}

Este trabajo trata del problema de optimizar el funcionamiento de un sistema de bombeo de agua con una estructura predefinida, con varios pozos y bombas, varios tanques y varios conductos y válvulas. El objetivo de la optimización es la minimización del coste operativo global. Este coste se relaciona con el coste energético individual de bombeo de cada pozo (kWh por metro cúbico) $\mathrm{y}$, especialmente, con la tarifa eléctrica que establece un precio diferente dependiendo del periodo. El sistema de control automático debe decidir qué válvulas y qué bombas deben ser operadas en cada momento a lo largo del día para minimizar el coste y cumplir con algunas restricciones.

La principal restricción es servir a cada tanque el caudal diario de agua requerido. Este caudal es variable en el tiempo e incierto, pero puede predecirse porque sigue un patrón diario aproximado. La otra limitación importante se debe al tamaño de los tanques, cada uno con un nivel máximo y mínimo que no debe ser superado.

Otras limitaciones secundarias que pueden aplicarse incluyen limitar de alguna forma el número de arranques y paradas. También la condición de que, dentro de lo posible, los niveles de los tanques sean lo más elevados posible para tener más margen en caso de avería.

\section{MODELADO MATEMÁTICO}

En primer lugar, se supone que el sistema de bombeo tiene $N_{p}$ bombas (o pozos), $N_{t}$ tanques y $N_{v}$ válvulas. Las válvulas se utilizan para reconfigurar el circuito hidráulico que conecta las bombas a los tanques. El número total de combinaciones posibles es $2^{N_{p}+N_{v}}$, pero no todas las combinaciones son posibles en un sistema dado. Definamos como $N_{c}$ el número de combinaciones válidas de válvulas y bombas. Con el fin de formular matemáticamente el problema, se define una matriz binaria, $M_{c}$, de tamaño $N_{c} \times\left(N_{p}+N_{v}\right)$, donde cada fila representa una de las combinaciones válidas y donde los elementos correspondientes toman el valor 1 ó 0 dependiendo del estado activo o inactivo de las válvulas y de las bombas en esa combinación.

A lo largo del documento, se utiliza un ejemplo de aplicación, obtenido de una instalación real de suministro de agua, para ilustrar el enfoque propuesto. El sistema de bombeo considerado tiene $N_{p}=3$ pozos (bombas), $N_{v}=2$ válvulas y $N_{t}=3$ tanques. La figura 1 muestra el esquema del sistema. El depósito 3 puede llenarse desde la bomba 1 , sea cual sea el estado de las válvulas, o de la bomba 2 , si la válvula 2 está abierta, o desde la bomba 3, también si la válvula 2 está abierta. El depósito 2 sólo puede llenarse desde la bomba 3 si la válvula 2 está cerrada y la válvula 1 abierta. Finalmente, el tanque 1 sólo puede llenarse desde la bomba 3 si ambas válvulas están cerradas. La bomba 1 y 3 pueden funcionar simultáneamente, pero la bomba 2 sólo puede funcionar si la bomba 3 está parada y la válvula 2 está abierta, porque no da altura suficiente para llenar los tanques 1 o 2. Teniendo en cuenta las limitaciones físicas

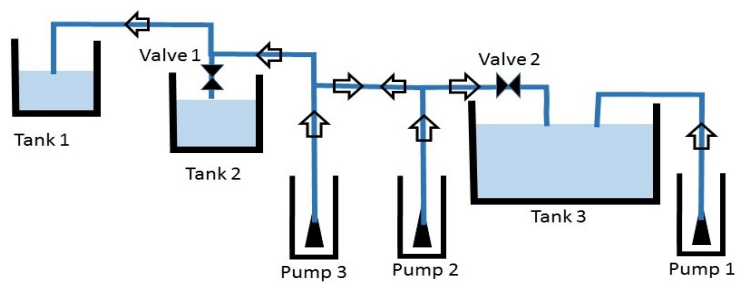

Figura 1: Esquema de la aplicación real.

descritas anteriormente, en la tabla 1 se muestra la matriz que define las combinaciones válidas de bombas y válvulas $N_{c}=10$. El valor $X$ representa que los caudales no dependen del estado de la válvula. Para cada combinación de válvulas y bombas, hay un caudal de salida resultante de cada bomba, y un caudal de entrada resultante para cada tanque. Esto puede expresarse mediante una matriz de caudales de bombeo, $F_{P}$, que tiene tantas columnas como combinaciones, y una fila por bomba, y una matriz de caudales de entrada 
Cuadro 1: Combinaciones válidas en el ejemplo de aplicación.

\begin{tabular}{|c|c|c|c|c|c|}
\hline Comb & $V_{1}$ & $V_{2}$ & $P_{1}$ & $P_{2}$ & $P_{3}$ \\
\hline 0 & $\mathrm{X}$ & $\mathrm{X}$ & 0 & 0 & 0 \\
1 & 0 & 0 & 0 & 0 & 1 \\
2 & 1 & 0 & 0 & 0 & 1 \\
3 & $\mathrm{X}$ & 1 & 0 & 0 & 1 \\
4 & $\mathrm{X}$ & 1 & 0 & 1 & 0 \\
5 & $\mathrm{X}$ & $\mathrm{X}$ & 1 & 0 & 0 \\
6 & 0 & 0 & 1 & 0 & 1 \\
7 & 1 & 0 & 1 & 0 & 1 \\
8 & $\mathrm{X}$ & 1 & 1 & 0 & 1 \\
9 & $\mathrm{X}$ & 1 & 1 & 1 & 0 \\
\hline
\end{tabular}

de tanque, $F_{T}$, que tiene tantas columnas como combinaciones y una fila por tanque, es decir, el tamaño de las matrices $F_{P}$ y $F_{T}$ es $\left(N_{p} \times N_{c}\right)$ y $\left(N_{t} \times N_{c}\right)$. En el ejemplo de aplicación, las matrices de caudales resultantes son $\left(\right.$ en $\left.m^{3} / h\right)$ :

$$
\begin{aligned}
& F_{P}=10\left[\begin{array}{cccccccccc}
0 & 0 & 0 & 0 & 0 & 20 & 20 & 20 & 20 & 20 \\
0 & 0 & 0 & 0 & 10 & 0 & 0 & 0 & 0 & 10 \\
0 & 8 & 10 & 12 & 0 & 0 & 8 & 10 & 12 & 0
\end{array}\right] \\
& F_{T}=10\left[\begin{array}{cccccccccc}
0 & 8 & 0 & 0 & 0 & 0 & 8 & 0 & 0 & 0 \\
0 & 0 & 10 & 0 & 0 & 0 & 0 & 10 & 0 & 0 \\
0 & 0 & 0 & 12 & 10 & 20 & 20 & 20 & 32 & 30
\end{array}\right]
\end{aligned}
$$

Siguiendo el mismo procedimiento, se puede formar una matriz $P$ con el consumo de energía eléctrica de cada bomba para cada combinación. La matriz tiene tantas columnas como combinaciones, y una fila por bomba, es decir, el tamaõ de la matriz $P$ es $\left(N_{p} \times N_{c}\right)$. En el ejemplo propuesto, la matriz de potencia (en $k W$ ) es:

$P=\left[\begin{array}{cccccccccc}0 & 0 & 0 & 0 & 0 & 12 & 12 & 12 & 12 & 12 \\ 0 & 0 & 0 & 0 & 5 & 0 & 0 & 0 & 0 & 5 \\ 0 & 13 & 11 & 7 & 0 & 0 & 13 & 11 & 7 & 0\end{array}\right]$

Para poder operar la instalación de suministro de agua, el sistema de gestión automática debe decidir cuál de esas combinaciones $N_{c}$ debe aplicarse en cada instante de tiempo. El objetivo natural es minimizar el coste total de operación. Para poder formular el problema de optimización, se podría definir una variable que tomara valores enteros de 0 a $N_{c}$. Sin embargo, con el fin de formular más fácilmente la función objetivo así como las restricciones, se propone un vector binario para definir la combinación aplicada en función del tiempo:

$$
\delta(t) \in\left\{\delta_{1}, \ldots, \delta_{N_{c}}\right\}
$$

donde

$$
\delta_{i}=[\underbrace{0 \cdots 0}_{i-1} \quad 1 \quad 0 \cdots 0.0]^{T}
$$

Por lo tanto, los elementos de $\delta(t)$ sólo pueden ser 0 o 1 , y sólo uno de los elementos puede ser diferente de cero (la suma de los elementos es 1).
Con estas definiciones, el vector que reúne los caudales de entrada de los tanques en un instante dado es simplemente el producto

$$
f_{T}(t)=F_{T} \delta(t)
$$

, el vector de caudales de salida de las bombas es

$$
f_{P}(t)=F_{P} \delta(t)
$$

, mientras que el vector de potencia consumida por las bombas es

$$
p(t)=P \delta(t)
$$

Para calcular el coste total, debe tenerse en cuenta la tarifa eléctrica. La tarifa puede expresarse como una función que define el precio en euro/ $k W h$ en función del tiempo, $T_{i}(t)$. Cada bomba podría tener una tarifa diferente, por lo tanto, definimos un vector fila como:

$$
T(t)=\left[\begin{array}{lll}
T_{1}(t) & \cdots & T_{N_{p}}(t)
\end{array}\right]
$$

Con esto, el coste total en un periodo de tiempo puede expresar

$$
J=\frac{1}{3600} \int T(t) P \delta(t) d t
$$

La ecuación de los tanques puede expresarse como:

$$
\dot{V}_{j}=f_{T, j}(t)-f_{O, j}(t)
$$

donde $f_{T, j}(t)$ y $f_{O, j}(t)$ son los caudales de entrada y salida del tanque $j$. Las ecuaciones de todos los tanques pueden unirse en forma matricial

$\dot{V}=\left[\begin{array}{c}\dot{V}_{1} \\ \cdots \\ \dot{V}_{N_{t}}\end{array}\right]=\left[\begin{array}{c}f_{T, 1}(t) \\ \cdots \\ f_{T, N_{t}}(t)\end{array}\right]-\left[\begin{array}{c}f_{O, 1}(t) \\ \cdots \\ f_{O, N_{t}}(t)\end{array}\right]=f_{T}(t)-f_{O}(t)$

Como no se conoce el caudal de salida futuro de cada tanque, $f_{O}(t)$, se debe utilizar una predicción $\hat{f}_{O}(t)$ para estimar la evolución del volumen. Por otra parte, el caudal de entrada puede expresarse como una función de $\delta(t)$, por lo tanto, la ecuación utilizada para estimar la evolución del volumen de los tanques (en $\left.m^{3} / s\right)$ es

$$
\dot{V}=\frac{1}{3600}\left(F_{T} \delta(t)-\hat{f}_{O}(t)\right)
$$

donde los caudales están en $m^{3} / h$.

Con el fin de obtener una formulación manejable del problema, las ecuaciones de tiempo continuo deben ser discretizadas. Si se elige un periodo de discretización constante $h$ (en segundos), las funciones vectoriales $T(t)$ y $\delta(t)$ serían entonces cambiadas por vectores de señales discretas $T[k]=T(t=K h)$ y $\delta[k]=\delta(t=k h) . \delta(t)$ se supone que mantiene un valor constante durante el intervalo $h$, es decir $\delta(t)=\delta[k]$ para 
$k h<=t<(k+1) h$. Teniendo en cuenta las unidades de las variables, el índice de coste (en euros) podría expresarse como:

$$
J=\frac{h}{3600} \sum T[k] P \delta[k]
$$

La ecuación de tiempo continuo de los tanques también debe discretizarse en el periodo $h$. La ecuación discretizada puede expresarse como:

$$
V[k+1]=V[k]+\frac{h}{3600}\left(F_{T} \delta[k]-\hat{f}_{O}[k]\right)
$$

con $V[k]=V(t=k h), \hat{f}_{O}[k]=\frac{1}{h} \int_{k h}^{(k+1) h} \hat{f}_{O}(t) d t$.

Las principales restricciones son los límites máximos y mínimos de los volúmenes de los tanques, por lo tanto, las restricciones pueden ser formuladas como

$$
V_{i, \min } \leq V_{i}[k] \leq V_{i, \max }, \quad i=1, \cdots, N_{t}
$$

o en forma matricial

$$
V_{\min } \leq V[k] \leq V_{\max }
$$

\section{PROBLEMA DE OPTIMIZACIÓN BÁSICO}

El problema básico de optimización consiste en minimizar el coste de la energía cumpliendo con las principales restricciones de mantener los volúmenes de todos los tanques dentro de su rango admisible (entre sus valores mínimo y máximo). Se debe añadir una restricción adicional para garantizar que los tanques terminen el día con el mismo volumen que han comenzado. De lo contrario, la solución óptima siempre terminará el día con los tanques completamente vacíos.

Teniendo en cuenta las ecuaciones introducidas en las secciones anteriores, con la definición de vectores $\delta[k], T[k], V[k]$ y $\hat{f}_{O}[k]$, horizonte de minimización $t_{m}=k_{m} h$, el problema de minimización puede ser formulado como:

$$
\begin{array}{cc}
\operatorname{mí}_{\delta} & \frac{h}{3600} \sum_{k=1}^{k_{m}} T[k] P \delta[k] \\
\text { s.t. } & \\
V_{\text {min }} \leq & V(0)+\frac{h}{3600} \sum_{j=1}^{k}\left(F_{T} \delta[j]-\hat{f}_{O}[j]\right) \leq V_{\text {max }} \\
& \sum_{j=1}^{k_{m}}\left(F_{T} \delta[j]-\hat{f}_{O}[j]\right) \geq 0 \\
\sum_{i=1}^{N_{c}} \delta_{i}[k]=1, \delta_{i}[k] \in\{0,1\} \\
k=1, \ldots, k_{m}
\end{array}
$$

$$
T[k]=\left\{\begin{array}{cl}
T(t=k h) & \text { if } \quad k \leq k_{m} \\
T\left(t=k_{m} h+\left(k-k_{m}\right) L h\right) & \text { if } \quad k>k_{m}
\end{array}\right.
$$

El problema de optimización (15) es mixto entero, donde las variables de decisión son los elementos del vector $\boldsymbol{\Delta}$ que sólo pueden tomar valores 0 o 1 . El número de variables de decisión es $N_{c} k_{m}$, y el

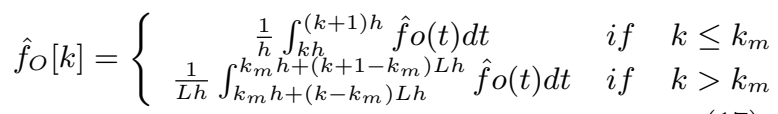

Se definen los siguientes vectores y matrices:

$$
\boldsymbol{\Delta}=\left[\begin{array}{c}
\delta[1] \\
\vdots \\
\delta\left[k_{m}\right]
\end{array}\right]_{k_{M} N_{c} \times 1}
$$

número de restricciones es $\left(2 N_{c}+2 N_{t}+1\right) k_{m}+N_{t}$. Para un horizonte de predicción normal de 1 día, si el número de variables es $1440 N_{c}$, y el número de te simples. Además, la optimización mixta entera sólo una vez al día), porque depende de la predicción del caudal futuro $\hat{f}_{O}[k]$, que es incierta, y que Por ejemplo, podría ejecutarse cada 10 minutos. obtenidos durante los primeros 10 minutos, destando el resto. Esta es una estrategia habitual Por lo tanto, con el fin de reducir la complejidad, carán realmente, proponemos utilizar dos periodos de discretización, uno corto para los primeros ins$h_{M}=L$ L. Además, el coste computacional, y el error de discretización en los grandes periodos, se propone utilizar vectoque los valores de los elementos de $\delta$ tomen cualquier valor real de 0 a 1 , pero con la restricción de durante una fracción del periodo largo, definida por el valor del elemento correspondiente de $\delta$. Esfija se aplica durante un periodo largo. Por otro lado, el coste computacional de la optimización se reduce, ya que el número de variables enteras se drásticamente.

parte, el problema de optimización se como $t_{m}=k_{m} h$, y el horizonte de tiempo total $t_{M}=t_{m}+\left(k_{M}-k_{m}\right) L h$, y se definen los vectores: En ese caso, sólo se aplicarán los valores de $\delta[k]$ 


$$
\begin{aligned}
& \boldsymbol{T}=\frac{h}{3600}\left[T[1] \cdots T\left[k_{m}\right] \quad T\left[k_{m}+1\right] L \cdots T\left[k_{M}\right] L\right] \\
& \boldsymbol{P}=\left[\begin{array}{cccc}
P & 0 & \cdots & 0 \\
0 & P & \ddots & \vdots \\
\vdots & \ddots & \ddots & \vdots \\
0 & \cdots & 0 & P
\end{array}\right]_{k_{M} N_{p} \times k_{M} N_{c}} \\
& \boldsymbol{F}_{k}=\left[\begin{array}{lll}
\underbrace{F_{T} \cdots F_{T}}_{k_{m}} & \underbrace{L F_{T} \cdots L F_{T}}_{k-k_{m}} & 0 \cdots 0
\end{array}\right]_{N_{t} \times k_{M} N_{c}} \\
& \boldsymbol{I}_{k}=\left[\begin{array}{lllll}
\underbrace{0 \cdots 0}_{(k-1) N_{c}} & \underbrace{1 \cdots 1}_{N_{c}} & 0 & \cdots & 0
\end{array}\right]_{1 \times k_{M} N_{c}} \\
& \hat{F}_{O}[k]=\sum_{j=1}^{k_{m}} \hat{f}_{o}[j]+L \sum_{j=k_{m}+1}^{k} \hat{f}_{o}[j]
\end{aligned}
$$

Con estas definiciones, el problema de minimización se puede formular como:

$$
\begin{aligned}
& \min _{\Delta} \boldsymbol{T P} \boldsymbol{\Delta} \\
& \text { s.t. } \\
& \boldsymbol{F}_{k} \boldsymbol{\Delta} \geq 3600 \frac{V_{\min }-V(0)}{h}+\hat{F}_{O}[k], k=1, \ldots, k_{M} \\
& \boldsymbol{F}_{k} \boldsymbol{\Delta} \leq 3600 \frac{V_{\max }-V(0)}{h}+\hat{F}_{O}[k], k=1, \ldots, k_{M} \\
& \boldsymbol{F}_{k_{M}} \boldsymbol{\Delta} \geq \hat{F}_{O}\left[k_{M}\right] \\
& \boldsymbol{I}_{k} \boldsymbol{\Delta}=1, \quad k=1, \ldots, k_{M} \\
& \boldsymbol{\Delta}[j] \in \begin{cases}0,1\} \in \boldsymbol{N}, \quad j=1, \cdots, k_{m} N_{c} \\
\boldsymbol{\Delta}[j] \in[0,1] \in \boldsymbol{R} \quad j=k_{m} N_{c}+1, \cdots, k_{M} N_{c}\end{cases}
\end{aligned}
$$

El número de variables de decisión enteras en el problema (24) es $N_{c} k_{m}$, y el número de variables de decisión reales es $N_{c}\left(k_{M}-k_{m}\right)$. El número de restricciones es $\left(2 N_{c}+2 N_{t}+1\right) k_{M}+N_{t}$.

\section{PROBLEMA DE OPTIMIZACIÓN AVANZADO}

El inconveniente principal del problema de optimización básico (24) es que la solución óptima tiende a implicar una gran cantidad de conmutaciones, es decir, las bombas y válvulas se detendrían y arrancarían cada pocos minutos. Este es un problema serio, ya que cada arranque y parada implica un desperdicio de energía y una reducción en la vida útil de los componentes hidráulicos. Por lo tanto, el problema de optimización debe ser modificado para lograr un número bajo de conmutaciones para que la propuesta sea útil.

Por otro lado, como estamos imponiendo un volumen final igual al inicial, el resultado de la optimización depende de la condición inicial de los tanques y del instante de tiempo en que se realiza la optimización. Además, desde un punto de vista práctico, es interesante que al menos una vez al día, los tanques tengan el máximo volumen almacenado posible para hacer frente a problemas inesperados a lo largo del día. Por lo tanto, proponemos imponer como un objetivo indirecto alcanzar los volúmenes máximos de tanque en un instante prefijado, que puede ser al final del periodo tarifario más barato (a las 8).

Tanto la limitación del número de conmutaciones como el forzado de unos volúmenes determinados en un instante se pueden abordar modificando el índice de coste o añadiendo restricciones.

\subsection{LIMITACIÓN DEL NÚMERO DE CONMUTACIONES}

Si consideramos el número de conmutaciones como el número de cambios en $\delta[k]$ a lo largo del periodo de optimización, necesitamos una ecuación que exprese ese número de cambios como una función de la matriz $\boldsymbol{\Delta}$. De hecho, como los valores de $\delta$ en los periodos más largos son reales, el número de conmutaciones sólo puede aplicarse a los periodos más cortos donde $\delta$ es entero. Si se definen las siguientes matrices:

$$
\begin{aligned}
I_{N}^{+} & =\left[\begin{array}{cccccc}
\overbrace{0}^{\cdots} & 0 & 1 & 0 & \cdots & 0 \\
\vdots & \ddots & \ddots & \ddots & \ddots & \vdots \\
0 & \ddots & \cdots & \ddots & 1 & 0 \\
0 & \cdots & \cdots & \cdots & 0 & 1
\end{array}\right]_{\left(k_{m}-1\right) N \times k_{m} N} \\
I_{N}^{-} & =\left[\begin{array}{cccccc}
1 & 0 & \cdots & \cdots & \cdots & 0 \\
0 & 1 & \ddots & \ddots & \ddots & 0 \\
\vdots & \ddots & \ddots & \ddots & \ddots & \vdots \\
0 & \cdots & 0 & 1 & \underbrace{0 \cdots}_{N}
\end{array}\right]_{\left(k_{m}-1\right) N \times k_{m} N}
\end{aligned}
$$

El número de cambios en $\delta[k]$ durante los $k_{m}$ periodos cortos se puede expresar como:

$$
\operatorname{sum}\left(\operatorname{abs}\left(Y_{N_{c}}\left[\delta_{a n t} ; \boldsymbol{\Delta}\left(1:\left(k_{m}-1\right) N_{c}\right)\right]\right)\right)
$$

La expresión anterior tiene en cuenta como un posible cambio el primer valor de $\Delta$ si éste es distinto del último del periodo anterior. $\delta_{a n t}$ no es una variable de decisión, sino un vector constante resultado de la optimización previa.

La primera alternativa para reducir el número de conmutaciones es incluir el número de cambios de $\delta$ en el índice de coste. En este caso, el problema 
de optimización tendría las mismas restricciones que (24), y solo se modificaría el índice de coste:

$J=J+\alpha_{c} \operatorname{sum}\left(\operatorname{abs}\left(Y_{N_{c}}\left[\boldsymbol{\delta}_{\text {ant }} ; \boldsymbol{\Delta}\left(1:\left(k_{m}-1\right) N_{c}\right)\right]\right)\right)$

El factor de ponderación $\alpha_{c}$ debe escogerse con cuidado para lograr un compromiso entre la minimización del coste y el número de conmutaciones.

En lo que respecta a limitar el número de conmutaciones introduciendo restricciones, aunque es posible, no es efectivo en la práctica. Supongamos que la optimización se realiza cada 10 minutos, y se limita el número de conmutaciones a $c_{\max }=2$. Entonces, en el peor caso, existe la posibilidad de que las 2 conmutaciones siempre ocurran en los primeros 10 minutos, resultando en un total de $2 \times 6 \times 24=288$ conmutaciones en un día.

\subsection{FORZADO DE VOLÚMENES}

Por otra parte, con el fin de forzar que los volúmenes alcancen un valor máximo en un instante determinado, la primera alternativa es agregar un término al índice de coste, con un factor de ponderación. Se propone definir un valor $k_{v}$, como el entero que representa el instante discreto más cercano al instante de tiempo cuando se requieren los volúmenes máximos. Si llamamos $t_{i}$ al tiempo inicial (en segundos) cuando se ejecuta la optimización, y $t_{v}$ el momento en que se requieren los volúmenes máximos, el valor de $k_{v}$ es

$k_{v}=\left\{\begin{array}{c}\operatorname{round}\left(\frac{t_{v}-t_{i}}{h}\right) \text { if } t_{v}-t_{i} \leq k_{m} h \\ k_{m}+\operatorname{round}\left(\frac{t_{v}-t_{i}-k_{m} h}{L h}\right) \text { if } t_{v}-t_{i}>k_{m} h\end{array}\right.$

Con esto, el índice de coste quedaría como

$$
J=J+\alpha_{V}\left(\hat{F}_{O}\left[k_{v}\right]-\boldsymbol{F}_{k_{v}} \boldsymbol{\Delta}+3600 \frac{V_{o b j}-V(0)}{h}\right)
$$

Donde $\alpha_{V}$ es un vector fila con el factor de ponderación para cada tanque, y $V_{o b j}$ es el vector de volúmenes que se alcanzará en el instante $t_{v}$.

El inconveniente del enfoque anterior es que el resultado es altamente dependiente del factor de ponderación $\alpha_{V}$ utilizado. La segunda alternativa consiste en agregar nuevas restricciones. La restricción a añadir para forzar que los volúmenes alcancen los valores requeridos en el tiempo especificado sería

$$
\boldsymbol{F}_{k_{v}} \boldsymbol{\Delta} \geq 3600 \frac{V_{o b j}-V(0)}{h}+\hat{F}_{O}\left[k_{v}\right]
$$

El inconveniente de este enfoque es que la restricción añadida puede provocar que el problema de optimización no sea factible. Esto puede pasar especialmente si el instante en que se ejecuta la optimización está cerca del instante en que se requieren los volúmenes máximos, es decir cuando $k_{v}$ es pequeño.

\subsection{PROBLEMA DE OPTIMIZACIÓN FINAL}

Se plantean 2 alternativas para el problema de optimización final, dependiendo de si los volúmenes máximos están incluidos en el índice de coste o se formulan como restricciones.

Se define:

$$
\begin{gathered}
J_{0}=\boldsymbol{T P} \boldsymbol{\Delta} \\
J_{c}=\alpha_{c} \operatorname{sum}\left(a b s\left(Y_{N_{c}}\left[\boldsymbol{\delta}_{\text {ant }} ; \boldsymbol{\Delta}\left(1:\left(k_{m}-1\right) N_{c}\right)\right]\right)\right) \\
J_{v}=\alpha_{V}\left(\hat{F}_{O}\left[k_{v}\right]-\boldsymbol{F}_{k_{v}} \boldsymbol{\Delta}+3600 \frac{V_{o b j}-V(0)}{h}\right)
\end{gathered}
$$

Con respecto a las restricciones, algunas son comunes a las dos alternativas:

$$
\begin{aligned}
\boldsymbol{F}_{k} \boldsymbol{\Delta} & \geq 3600 \frac{V_{\min }-V(0)}{h}+\hat{F}_{O}[k], k=1, \ldots, k_{M} \\
\boldsymbol{F}_{k} \boldsymbol{\Delta} & \leq 3600 \frac{V_{\max }-V(0)}{h}+\hat{F}_{O}[k], k=1, \ldots, k_{M} \\
\boldsymbol{F}_{k_{M}} \boldsymbol{\Delta} & \geq \hat{F}_{O}\left[k_{M}\right] \\
& \boldsymbol{I}_{k} \boldsymbol{\Delta}=1, \quad k=1, \ldots, k_{M} \\
& \boldsymbol{\Delta}[j] \in \begin{cases}0,1\} \in \boldsymbol{N}, \quad j=1, \cdots, k_{m} N_{c} \\
\boldsymbol{\Delta}[j] \in[0,1] \in \boldsymbol{R} \quad j=k_{m} N_{c}+1, \cdots, k_{M} N_{c}\end{cases}
\end{aligned}
$$

Mientras que la posible restricción adicional es:

$$
C_{v}:=\boldsymbol{F}_{k_{v}} \boldsymbol{\Delta} \geq 3600 \frac{V_{o b j}-V(0)}{h}+\hat{F}_{O}\left[k_{v}\right]
$$

Con esto, los posibles problemas de optimización pueden resumirse como

1. Minimizar $J_{0}+J_{c}$ sujeto a las restricciones comunes más $C_{v}$.

2. Minimizar $J_{0}+J_{c}+J_{v}$ sujeto a las restricciones comunes.

Teniendo en cuenta las ventajas e inconvenientes, se propone utilizar el problema 1 para garantizar volúmenes máximos de los tanques en el momento especificado, pero cambiar al problema 2 en caso de que el problema 1 sea no factible.

\section{EJEMPLO DE APLICACIÓN}

Se utilizará el ejemplo de aplicación descrito en la sección 3, obtenido de una instalación real de suministro de agua, para ilustrar el enfoque propuesto. Las tarifas de las tres bombas se muestran en la tabla 2. Para realizar los cálculos, el caudal de salida de los tanques se simula por medio de una serie de Fourier ajustada a partir de datos reales, a la que se agrega un término aleatorio filtrado para simular variaciones inciertas. Para la predicción del caudal de salida, se ha utilizado un 
Cuadro 2: Tarifas de las tres bombas en el ejemplo de aplicación (en eur/kWh).

\begin{tabular}{|c|c|c|c|}
\hline Periods & Pump 1 & Pump 2 & Pump 3 \\
\hline $0<t<480$ & 0.063 & 0.066 & 0.066 \\
$480<t<540$ & 0.079 & 0.088 & 0.088 \\
$540<t<600$ & 0.1 & 0.088 & 0.088 \\
$600<t<900$ & 0.1 & 0.099 & 0.099 \\
$900<t<960$ & 0.079 & 0.099 & 0.099 \\
$960<t<1440$ & 0.079 & 0.088 & 0.088 \\
\hline
\end{tabular}

algoritmo simple que predice para el día siguiente el mismo caudal horario medido en el día de hoy.

Los problemas de optimización final descritos en la sección 5.3 se han resuelto con Matlab, utilizando Yalmip como parser y el solver CBC.

Los volúmenes mínimos permitidos para los tanques son $V_{\min }=[100 ; 100 ; 740]$ y los volúmenes máximos $V_{\max }=[500 ; 500 ; 3700]$, en $\mathrm{m}^{3}$. Con el fin de controlar el sistema de bombeo, la optimización se realiza cada 10 minutos, con un horizonte de 24 horas, con un periodo corto de $h=60$ segundos, con $k_{m}=30$ y $k_{M}=47$ (es decir $\mathrm{L}=30$ ). Sólo se aplican las 10 primeras órdenes de bombeo resultantes de la optimización.

En el instante $t_{v}=480 \cdot 60$ segundos, es decir, al final del periodo más barato, se requiere que los volúmenes de los tanques sean cercanos a los máximos, $V_{o b j}=[492 ; 492 ; 3640]$.

Se utiliza siempre el problema de optimización 1, donde los volúmenes en $t_{v}$ son una restricción, y el número de conmutaciones se incluye en el índice de coste, con un factor de ponderación $\alpha_{c}$, excepto cuando el problema no resulta factible, en que se resuelve el problema 2 en su lugar (con ambos términos en el índice de coste, con $\left.\alpha_{v}=0,001\right)$.

La figura 2 muestra la evolución de los volúmenes de los tanques, los caudales de las bombas y el estado de las válvulas durante 3 días de simulación. En este caso, el número de conmutaciones no se considera (es decir, $\alpha_{c}=0$ ). El resultado es un gran número de conmutaciones (más de 1000).

Las figuras 3, 4 y 5 muestran el resultado de la simulación para diferentes valores de $\alpha_{c}$. Cuanto mayor es el valor de $\alpha_{c}$, menor es el número de conmutaciones diario, pero mayor el coste $\left(n_{\text {con }}=\right.$ $20, J=54$ para $\alpha_{c}=0,01, n_{\text {con }}=12, J=55$ para $\alpha_{c}=0,1, n_{\text {con }}=8, J=59$ para $\left.\alpha_{c}=0,5,\right)$.

Observando la evolución de los volúmenes (mostrada como un porcentaje de su capacidad máxima), la simulación comienza a las 0 horas con los tres tanques en el centro de su rango. A las 8 no se pueden conseguir los volúmenes $V_{o b j}$, pero después

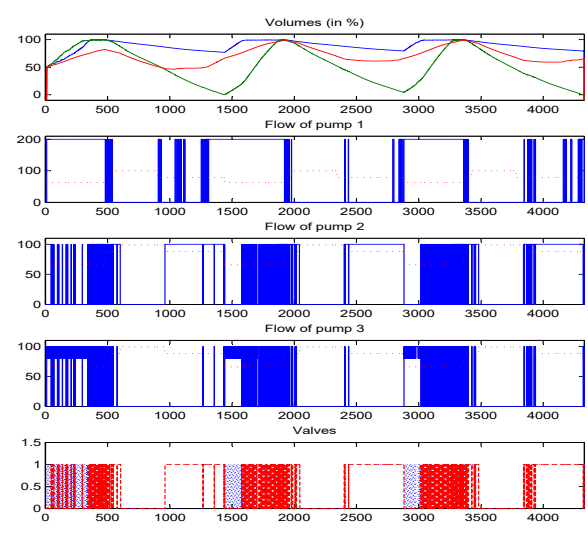

Figura 2: Resultados de la simulación para $\alpha_{c}=0$. Volúmenes (en porcentaje), caudal de las bombas 1,2 y 3, y estado de las válvulas.

de 24 horas, se llega a un gráfico de volumen cuasi periódico en el que sí se consiguen. Las figuras muestran los caudales de las bombas junto con el precio horario de la energía según la tarifa. Se observa que una vez que el patrón cuasi periódico se establece, las bombas nunca se ponen en marcha en los periodostarifarios más caros.
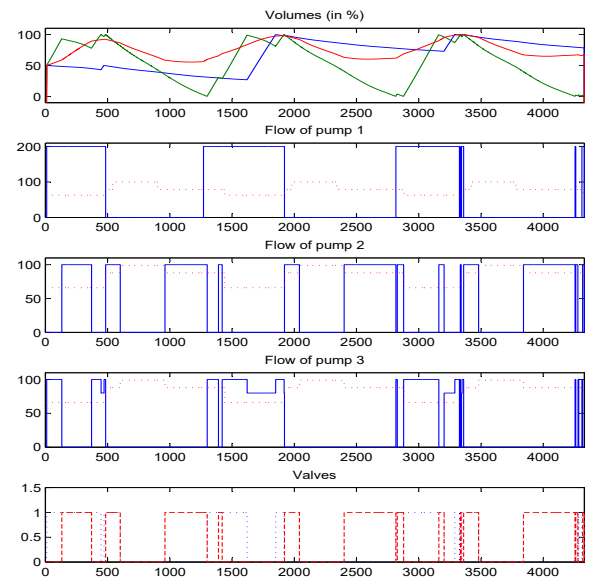

Figura 3: Resultados de la simulación para $\alpha_{c}=$ 0,01 . Volúmenes (en porcentaje), caudal de las bombas 1,2 y 3 , y estado de las válvulas.

\section{CONCLUSIONES}

En este trabajo se ha abordado la optimización de la operación de un sistema de bombeo de agua por medio de solvers estándar. El objetivo es determinar qué válvulas y bombas deben estar activas en cada instante de tiempo para minimizar el coste de operación, teniendo en cuenta los periodos de 

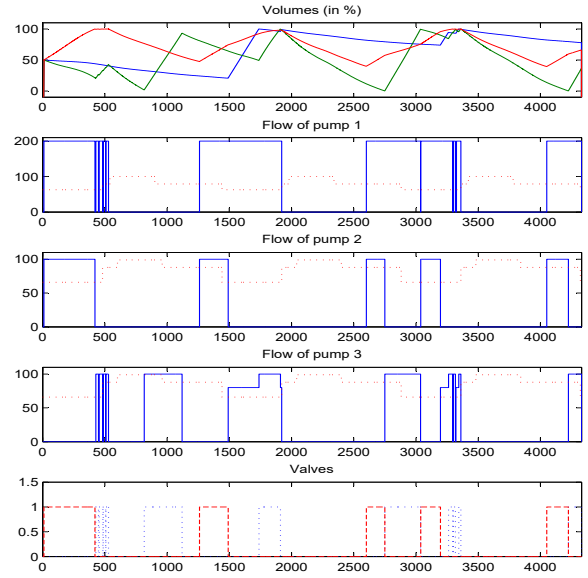

Figura 4: Resultados de la simulación para $\alpha_{c}=$ 0,1 . Volúmenes (en porcentaje), caudal de las bombas 1,2 y 3 , y estado de las válvulas.

tarificación. Las restricciones principales son los volúmenes máximo y mínimo de los tanques. Se propone un modelo matemático del problema con el fin de formular, en forma matricial, el índice de coste y las restricciones, para poder usar solvers estándar como CBC.

Se trata de un problema de optimización mixta entera, que tiene un coste computacional elevado. Para reducir el coste, teniendo en cuenta que la optimización se va a resolver cada poco tiempo, y que solo se van a aplicar los primeros valores obtenidos, se propone reducir el número de variables enteras, sustituyéndolas por variables reales a partir de un instante determinado en el horizonte de optimización. Se tiene en cuenta el número de conmutaciones en el índice de coste, ya que en caso contrario el número de conmutaciones resultante es muy elevado. Se analiza un ejemplo de un sistema de suministro de agua real, con 3 tanques, 3 bombas y 2 válvulas, para demostrar la validez del enfoque, utilizando Yalmip como parser y CBC como solver. La propuesta planteada permite resolver los problemas de optimización en pocos segundos en un ordenador personal de sobremesa.

\section{Agradecimientos}

Financiado por el proyecto TEC2015-69155-R del Ministerio de Economía y Competitividad.

\section{Referencias}

[1] Bunn, S. and Reynolds, L. (2009). The energy-efficiency benefits of pump scheduling optimization for potable water supplies. IBM Journal of Research and Development, 53:5:1 - 5:13. DOI:10.1147/JRD.2009.5429018.
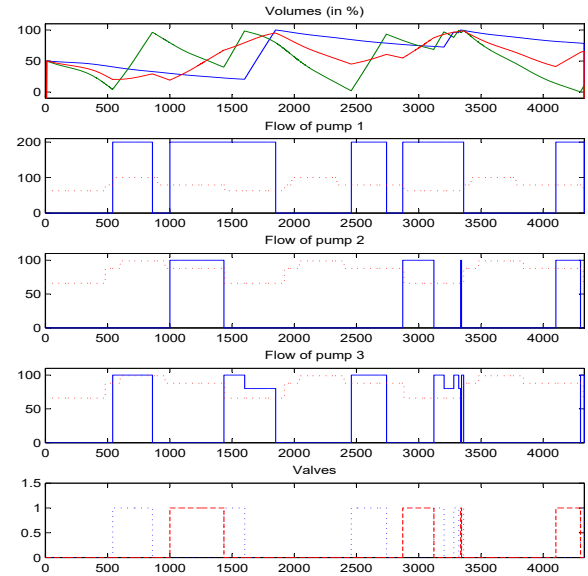

Figura 5: Resultados de la simulación para $\alpha_{c}=$ 0,5 . Volúmenes (en porcentaje), caudal de las bombas 1, 2 y 3, y estado de las válvulas.

[2] Fang, H., Zhang, J., and liang Gao, J. (2010). Optimal operation of multi-storage tank multi-source system based on storage policy. Journal of Zhejiang University-SCIENCE A, 11:571-579. DOI:10.1631/jzus.A0900784.

[3] Ormsbee, L., Lingireddy, S., and Chase, D. (2009). Optimal Pump Scheduling For Water Distribution Systems. In Proceedings of the $4^{\text {th }}$ Multidisciplinary International Conference on Scheduling : Theory and Applications (MISTA 2009), 10-12 August 2009, Dublin, Ireland, pages $341-356$.

[4] Ormsbee, L. E. and Lansey, K. E. (1994). OPTIMAL CONTROL OF WATER SUPPLY PUMPING SYSTEMS. Journal of Water Resources Planning and Management, 120:237252. ISSN:0733-9496/94/0002- 0237.

[5] Pasha, M. F. K. and Lansey, K. (2009). Optimal Pump Scheduling by Linear Programming. In Proceedings of the $4^{\text {th }}$ World Environmental and Water Resources Congress 2009 May 17-21, 2009 - Kansas City, Missouri, United States, pages 341-356.

[6] Powell, R. S. and McCormick, G. (2004). Derivation of NearOptimal Pump Schedules for Water Distribution by Simulated Annealing. J. Operational Res. Soc, 55:728-736.

[7] Sotelo, A., Lücken, C., and Barán, B. (2002). Multiobjective Evolutionary Algorithms in Pump Scheduling Optimization. In Proceedings of the Third International Conference on Engineering Computational Technology, Stirling, Scotland., page 175-176. 